\title{
Routing in SONET/VCAT based Optical WDM Networks (Invited Paper)
}

\author{
Kevin Yang ${ }^{1}$ and Krishna Sivalingam ${ }^{1,2}$ \\ ${ }^{1}$ Dept. of CSEE, University of Maryland, Baltimore County, Baltimore, MD 21250 \\ ${ }^{2}$ Dept. of CSE, Indian Institute of Technology Madras, Chennai, INDIA \\ Email: \{kevinryang@gmail.com, krishna@umbc.edu\}
}

\begin{abstract}
In this paper, we investigate problems related to optical wavelength division multiplexing (WDM) networks that use the virtual concatenation (VCAT) mechanism of synchronous optical network (SONET) technology. VCAT, an end-to-end mechanism, allows SONET based optical WDM networks to carry traffic in finer granularity via multiple paths or a single path with multiple wavelengths. The benefits include efficient utilization of link capacity and implicit partial link failure protection if link-disjoint paths are used. However, multi-path routing causes differential delay among constituent paths and requires high-speed buffer capacity at receiving nodes. In this paper, we propose four route selection methods to study the tradeoffs of VCAT's traffic splitting feature. The performance analysis is conducted using a discrete-event simulation model. Optical WDM grooming networks under dynamic traffic demand are considered. The simulation results quantify the costs and benefits of using the VCAT mechanism in SONET based optical WDM networks. The simulation results confirm the benefits of carrying traffic in finer granularity. MP and SPMW consistently outperform the traditional SPSW method by 50 percent and 90 percent respectively in blocking performance. In the event of a link failure, the implicit protection ratio increases as connections use more link-disjoint paths. The results also show that differential delay, which is the cost of VCAT's multi-path routing, increases as the number of link-disjoint paths increases.
\end{abstract}

\section{INTRODUCTION}

This paper considers optical Wavelength division multiplexing (WDM) networks [1] that use Synchronous optical network (SONET) as the underlying layer [2]. In particular, we consider the Virtual concatenation (VCAT) method, that was designed to address the inefficiencies of the traditional SONET concatenation mechanism [3]. This technique can split requested connection bandwidth into a set of traffic substreams and route them across the network via multiple paths. The benefits of multi-path routing include efficient utilization of link capacity, more evenly distributed traffic load, and implicit partial link failure protection if paths are link-disjoint [4].

When multiple paths are used to route a connection, the difference between the longest and shortest sub-stream path delay is called as the differential delay. The presence of differential delay requires the receiving node to store information until data from all constituent paths reach the destination. The buffer capacity increases as transmission rates and differential delay increase. For instance, in an optical WDM network with 16 wavelengths per link and a wavelength capacity of OC48 (2.5 gigabits per second), an average differential delay of
10 milliseconds requires 50 megabytes of buffer capacity per link. As link bandwidths increase, the buffer requirements also increase.

This paper aims to quantify the costs and benefits of VCAT's traffic splitting feature. We consider four route selection methods to study the tradeoffs: single-path-singlewavelength (SPSW), multi-path (MP), single-path-multiwavelength (SPMW), and single-path-multi-wavelength-multipath (SPMW-MP). The SPSW method routes a connection's traffic sub-streams using the same wavelength on a single path; the MP method routes a connection's traffic sub-streams using as many link-disjoint paths as possible; the SPMW method routes a connection's traffic sub-streams using multiple wavelengths on a single path; and the SPMW-MP method is a hybrid of SPMW and MP. While single-path methods avoid the differential delay problem, they also forgo the potential benefits of routing with multiple paths mentioned earlier.

A discrete-event simulator is used for performance analysis. We consider optical WDM grooming networks under dynamic traffic demand. Performance evaluation metrics include blocking probability, link utilization, connection setup time, differential delay, and implicit link failure protection ratio. The simulation results confirm the benefits of carrying traffic in finer granularity. MP and SPMW consistently outperform the traditional SPSW method by 50 percent and 90 percent respectively in blocking performance. In the event of a link failure, the implicit protection ratio increases as connections use more link-disjoint paths. The results also show that differential delay, which is the cost of VCAT's multi-path routing, increases as the number of link-disjoint paths increases.

\section{BACKGROUND AND RELATED WORK}

This section provides a brief description of SONET, VCAT, survival techniques in optical WDM networks, and other work related to this paper.

\section{A. Optical WDM networks}

Wavelength division multiplexing (WDM) is a technology which can send multiple light beams of different wavelengths simultaneously through an optical fiber [1], [2]. Technology demonstrations have shown that more than 100 wavelengths are possible, with each wavelength operating at up to $40 \mathrm{Gbps}$. WDM based optical backbone networks can be developed using optical crossconnects that support wavelength routing. 


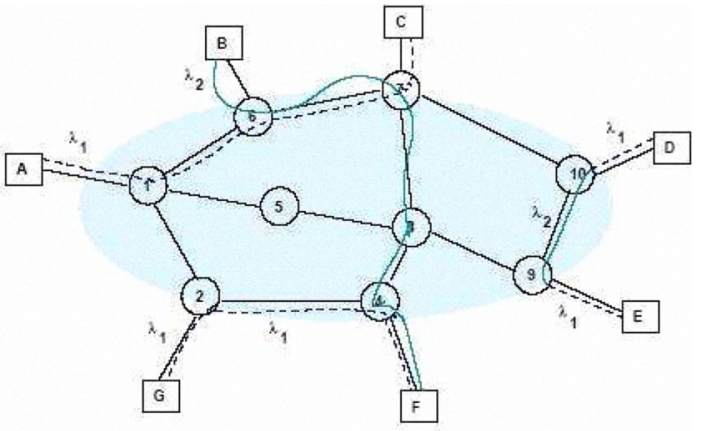

Access Station

Switching node (Photonic switch)

$\lambda_{1}$

$x_{2}$

Fig. 1. A wavelength-routed optical WDM network

Wavelength routing in optical WDM networks allows network nodes to communicate with each other via all-optical lightpaths [5]. Figure 1 shows a typical wavelength-routed optical WDM network where optical routing nodes are interconnected by fiber links. Traffic is carried over a lightpath between two nodes is an all-optical manner, without any intermediate optical-to-electronic conversion [1]. Wavelength converters, that optically convert data on one wavelength to another, can also be available at intermediate optical crossconnects. These devices help eliminate the wavelength continuity constraint while establishing lightpaths.

On each of the wavelengths, data can be carried over SONET or ITU G.709 based framing. In this work, we assume that SONET based framing is provided. Also, since each channel bandwidth is $10 \mathrm{Gbps}$ and higher, it is often not the case that a single connection will be alloted an entire wavelength. Hence, multiple lower bit-rate streams will be allotted to a wavelength, in order to improve link utilization. This technique is referred to as traffic grooming [6].

\section{B. SONET/VCAT}

SONET has been a successful standard for communicating digital information over optical fiber [7]. Virtual Concatenation (VCAT) is an inverse multiplexing technique [8] used to split a connection into a set of traffic sub-streams, which can be routed independently across the network. It was proposed as a part of the next-generation SONET to address the inefficiency of the traditional concatenation method - contiguous concatenation (CCAT) [4]. In CCAT, concatenation functionality is required at source, destination, and all intermediate nodes, which could be expensive to implement. However, in VCAT, only the source and the destination require concatenation functionality. Since the sizes of concatenated payload containers in CCAT are fixed, a large amount of bandwidth could be wasted. For example, routing a Gigabit Ethernet connection within a concatenated OC-48 (2.5 Gbps) channel leads to a
60 percent bandwidth waste. VCAT solves this problem by allowing concatenated payload containers of arbitrary sizes. Fig. 2 shows an example of using SONET VCAT to admit a new STS-3c connection that requires three OC-1 slots. Since the OC-12 signal on lightpath $A$ has only two free slots, VCAT allows the incoming STS-3c request to be split and routed across lightpath $A$ and a new lightpath $B$.
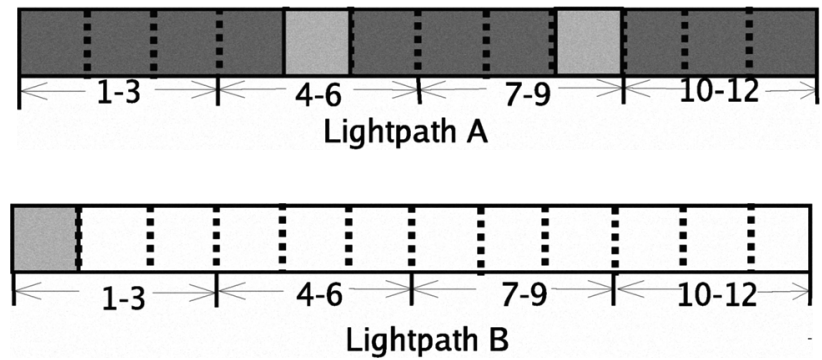

Fig. 2. An example of using SONET VCAT to carry a STS-3c connection.

VCAT enables SONET based optical WDM networks to carry traffic in finer granularity and to efficiently utilize link capacity [3]. When the network is congested, the ability to split the bandwidth over multiple paths can significantly increase the number of connection requests admitted. In addition, multipath routing allows traffic load to be more evenly distributed in the network and provides implicit partial link failure protection if paths are link-disjoint. However, VCAT is subjected to the problem of differential delay. For a connection that uses multiple paths, the difference between its longest and shortest path delay is known as the differential delay. For example, assume that connection $\mathrm{N}$ uses two paths and their delays are 10 milliseconds and 15 milliseconds respectively, then N's differential delay is 5 milliseconds. The disadvantage of differential delay is the requirement of installing high-speed buffer capacity at the receiving node to store information until data from all constituent paths reach the destination. The size of buffer required could be substantial as transmission rate and differential delay increase. Hence, it is essential to design network routing mechanisms that balance the benefits of bandwidth efficiency with the costs of differential delay. This forms the main focus of this paper.

One advantage of splitting a traffic into several sub-streams is the inherent protection from link failures. Thus, if any of the sub-streams fails, the connection can continue to operate, although with reduced bandwidth until the failed link is fixed.

The following sections present recent work closely related to this paper.

\section{Related Work}

The benefits of SONET VCAT on optical WDM grooming networks were explored in [3]. The authors have quantitatively demonstrated that VCAT can improve bandwidth efficiency, simplify network control, and balance network load. In our paper, we investigate VCAT's effect on link capacity utilization and link failure protection in great detail. 
In [9], the authors considered the problem of minimizing the differential delay in a virtually concatenated Ethernet over SONET system by suitable path selection. Related work on this problem is also presented in [10]. In [11], the authors present a widest path first heuristic and an integer linear programming based method for multi-path establishment, based on a cumulative differential delay definition. In [12], the authors present heuristics for setting up multiple paths while meeting differential delay constraints. In order to keep our heuristic simple, we do not attempt to minimize differential delay during the path selection process.

In [13], [14], the authors considered an explicit partial link failure protection mechanism where each connection has a primary path and a backup path. The backup bandwidth is a fraction of the primary bandwidth. In case of a link failure, the connection is carried on the backup path with reduced capacity and the system will try to identify additional capacity on the same backup path. In this paper, we only consider the implicit partial link failure protection provided by VCAT's multi-path routing and we do not attempt to find additional capacity when link failure occurs.

In [15], the authors present an effective multipath bandwidth metric while meeting a connection's reliability requirement. The paper also presents heuristics for setting up multi-path connections, while considering cost and reliability.

\section{Routing AND TRAFFic SplitTing AlgorithmS}

This section explains the route selection methods used to study the tradeoffs of SONET VCAT's traffic splitting feature. In the following subsections, $s$ denotes a given connection request's source node, $d$ the destination node, $b$ the requested connection bandwidth, $p$ the maximum number of link-disjoint paths between $s$ and $d$, and $w$ the maximum number of wavelengths that connections can use on a path. In the discussions below, it is assumed the minimum granularity of assignment is OC-1; this may be generalized to OC-3, etc. depending upon the optical switch's limitations.

\section{A. Single-Path-Single-Wavelength (SPSW)}

VCAT can route a connection across the network using either a single path or multiple paths. The usage of multiple paths would result in differential delay since different paths are likely to have different delays. The simplest way to eliminate differential delay is to route the connection using only one path and one wavelength. We define single-path-single-wavelength (SPSW) as a method that routes all SONET VCAT traffic substreams of a connection using the same wavelength on a single path. SPSW is the traditional route selection method and its heuristic works as follows:

- Step 1: find the shortest path between $s$ and $d$ such that each link on the path has one or more wavelengths with free capacity of at least $b$. If no such path exists, then the connection request is blocked and the algorithm ends.

- Step 2: select appropriate wavelengths for the lightpath. Without wavelength conversion, the establishment of a lightpath requires the usage of the same wavelength on all links. With wavelength conversion, different wavelengths can be used. If a lightpath can not be established, then the connection request is blocked and the algorithm ends.

- Step 3: update the available capacity of links along the path.

In Fig. 3, an OC-10 connection is routed using the SPSW method. The entire connection bandwidth is carried on $\lambda_{0}$ because of the single wavelength and single path constraints.

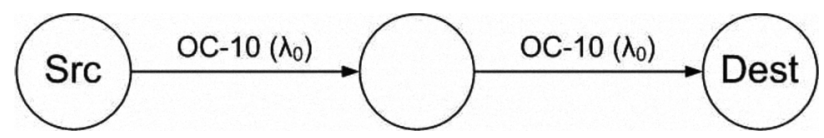

Fig. 3. Routing an OC-10 connection using the single-path-single-wavelength (SPSW) method.

\section{B. Single-Path-Multi-Wavelength (SPMW)}

With VCAT, single-path routing can be achieved using either a single wavelength or multiple wavelengths. We define single-path-multi-wavelength (SPMW) as a method that routes all SONET VCAT traffic sub-streams of a connection using different wavelengths on a single path. The heuristic works as follows:

- Step 1: evenly divide $b$ into $w$ component sub-streams with OC-1 as the basic unit. Examples are listed in Table I.

- Step 2: find a path for the first traffic sub-stream using the SPSW route selection heuristic described earlier.

- Step 3: if a path is found, then find a separate wavelength on the same path to satisfy each of the remaining traffic sub-streams. Otherwise, the connection request is blocked.

- Step 4: if the remaining traffic sub-streams can not be routed on the same path using different wavelengths and $w$ is greater than 1 , then decrement $w$ by 1 and repeat Step 1,2, and 3. Otherwise, update the available capacity of links along the path.

In Fig. 4, an OC-10 connection is routed using the SPMW method. The connection bandwidth is carried on a single path using three different wavelengths. The OC-4 traffic sub-stream is carried on $\lambda_{0}$, the OC-3 traffic sub-streams are carried on $\lambda_{1}$ and $\lambda_{2}$.

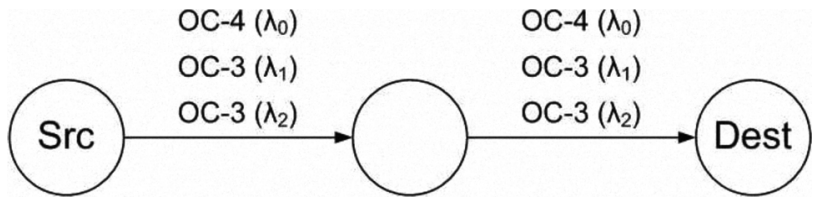

Fig. 4. Routing an OC-10 connection using the single-path-multi-wavelength (MP) method. 


\section{Multi-Path (MP)}

Routing traffic across the network via multiple paths allows efficient utilization of link capacity and more evenly distributed traffic load. In addition, connections receive implicit partial link failure protection if paths are link-disjoint. We define multi-path (MP) as a method that routes SONET VCAT traffic sub-streams of a connection using as many link-disjoint paths as possible where the requested bandwidth is divided equally among all paths. The heuristic works as follows:

TABLE I

BANDWIDTH SPLITTING EXAMPLES

\begin{tabular}{||l|c|l||}
\hline \hline Bandwidth & \# Link-Disjoint Paths & Sub-streams \\
\hline \hline OC-1 & 3 & OC-1 \\
\hline OC-2 & 3 & OC-1, OC-1 \\
\hline OC-9 & 3 & OC-3, OC-3, OC-3 \\
\hline OC-10 & 3 & OC-4, OC-3, OC-3 \\
\hline OC-11 & 3 & OC-4, OC-4, OC-3 \\
\hline \hline
\end{tabular}

- Step 1: find $p$, which is the minimum of two values: the number of outgoing links at $s$ and the number of incoming links at $d$.

- Step 2: evenly divide $b$ into $p$ component sub-streams with $\mathrm{OC}-1$ as the basic unit. Examples are listed in Table I.

- Step 3: find a path for each traffic sub-stream using the SPSW route selection heuristic described earlier such that all paths are link-disjoint.

- Step 4: if the number of paths found is less than the number of traffic sub-streams and $p$ is greater than 1 , then decrement $p$ by 1 and repeat Step 2 and 3 .

- Step 5: if the number of paths found is equal to the number of traffic sub-streams, then calculate the differential delay and update the available capacity of links along all paths. Otherwise, the connection request is blocked.

In Fig. 5, an OC-10 connection is routed using the MP method. The connection bandwidth is carried on three linkdisjoint paths. The OC- 4 traffic sub-stream is routed on the one-hop path using $\lambda_{0}$, the OC-3 traffic sub-streams are routed on the zero-hop path using $\lambda_{1}$ and the two-hop path using $\lambda_{0}$.

\section{Single-Path-Multi-Wavelength-Multi-Path (SPMW-MP)}

We next define a hybrid route selection method that combines SPMW and MP as single-path-multi-wavelength-multipath (SPMW-MP). This method first tries to establish a connection using the SPMW method and if SPMW fails, it uses the MP method. We expect SPMW-MP to admit more connection requests than SPMW and MP because it tries both single-path and multi-path approaches during connection setup.

The single-path methods avoid the differential delay problem. However, they forgo the potential benefits of routing with multiple paths. The complexity of these route selection methods are dominated by the running time of Dijkstra's algorithm, which is a part of RWA. With a linear storage implementation, Dijkstra's algorithm has a complexity of $O\left(n^{2}+m\right)$ where $n$

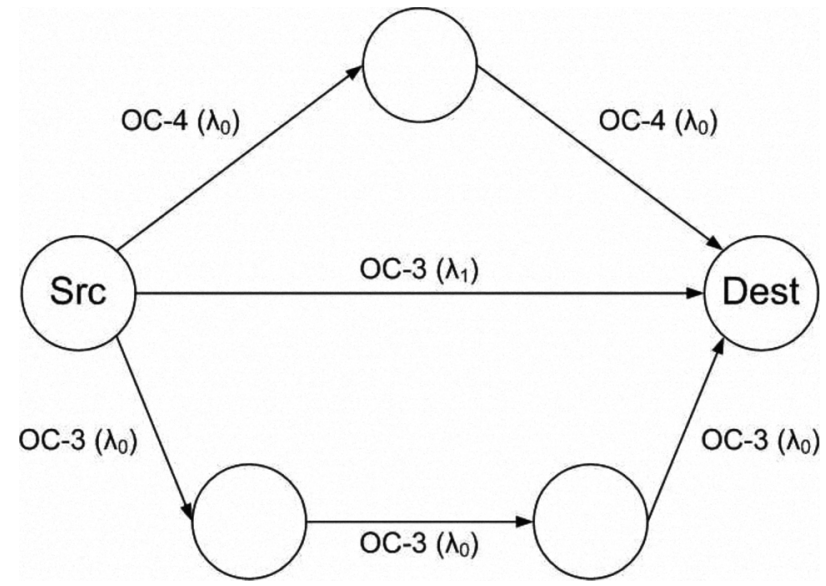

Fig. 5. Routing an OC-10 connection using the multi-path (MP) method.

is the number of nodes and $m$ is the number of links. SPSW is the simplest method because it only has to perform RWA once in all cases. SPMW is more computationally intensive than SPSW as it has to perform RWA $w$ times in the worst case. The MP method has to perform RWA $p$ times in the best case and $p(p+1) / 2$ times in the worst case. Thus, MP is the most computationally expensive method, as implemented.

\section{Performance Analysis}

The section presents the simulation model used for performance analysis and the performance of the route selection methods.

a) Simulation Model: The algorithms were implemented in a discrete-event simulator using the YACSIM library [16] and made the following assumptions in our model:

- A network with dynamic traffic demand where connections arrive and leave the network, one at a time, following a Poisson arrival process and negative-exponentialdistribution duration.

- The selection of source and destination for each connection request is based on a uniform distribution. The source is first selected from the set of all possible nodes, and then the destination is selected from the set of all other nodes.

- Routing paths are determined at the time of connection request arrival.

- The Erlang load offered is adjusted for each simulation experiment to keep the blocking probability between 0 and 0.1 .

- There are 16 wavelengths per link and the first-fit algorithm is used for wavelength assignment.

- All wavelengths have the same capacity of $\mathrm{T}=\mathrm{OC}$ 48 (2.5 Gbps) and the bandwidth requested by each connection $\left(\mathrm{d}_{m}\right)$ is uniformly distributed with a mean of $\mathrm{G}_{a v}[14]$.

$$
\begin{aligned}
d_{m} \sim \text { uniform }\left(1,2 G_{a v}-1\right) & 1 \leq G_{a v} \leq T / 2 \\
d_{m} \sim \operatorname{uniform}\left(2 G_{a v}-T, T\right) & T / 2<G_{a v} \leq T
\end{aligned}
$$


For example, a $\mathrm{G}_{a v}$ of 18 implies that $\mathrm{d}_{m} \sim$ uniform(1, $35), \mathrm{a}_{a v}$ of 24 implies that $\mathrm{d}_{m} \sim$ uniform $(1,47)$, and a $\mathrm{G}_{a v}$ of 30 implies that $\mathrm{d}_{m} \sim$ uniform $(12,48)$.

- Link failures are introduced, one at a time, after 10,000 connection requests are made. The arrival process is modeled as a Poisson process with a rate of $0.015 \lambda$, which guarantees at least 1 link failure per 100 connection requests. However, we limit the total number of link failures to 1 per 100 connection requests.

- For the SPMW route selection method, the maximum number of wavelengths per connection is limited to 4 .

The main simulation parameters and their values are listed in Table II.

TABLE II

SiMULATION PARAMETERS AND VALUES

\begin{tabular}{|l|l|}
\hline Simulation Parameters & Values \\
\hline \hline \# Requests per simulation & $1,000,000$ \\
\hline \# wavelengths per link & 16 \\
\hline Wavelength capacity & OC-48 $(2.5 \mathrm{Gbps})$ \\
\hline Average connection bandwidth $\left(\mathrm{G}_{a v}\right)$ & $\mathrm{OC}-12,18,24,30$ \\
\hline Connection arrival rate $(\lambda)$ & $10-30$ \\
\hline Mean connection duration $(1 / \mu)$ & 10 \\
\hline Link failure arrival rate & $0.015 \lambda$ \\
\hline Mean link failure duration & 1 \\
\hline Propagation delay per kilometer & $5 \mu \mathrm{s}$ \\
\hline Processing delay per node & $100 \mu s$ \\
\hline Network topology & NSFNET, USANET \\
\hline Max \# wavelengths per connection & 4 \\
\hline
\end{tabular}

b) Performance Metrics: The performance metrics considered are:

- Blocking Probability: is defined as the number of connection requests rejected over the total number of connection requests made, during the simulated time interval.

- Link Utilization: is defined as the percentage of total link capacity used at specific instances of a simulation experiment. A measurement is taken every 10,000 connection requests and the average is reported.

- Setup Time: is defined as the amount of time needed to establish a connection request. This includes time to compute routing paths and to update the available capacity of links along all paths.

- Differential Delay: is defined as the difference between the longest and shortest delay of paths carrying substreams of a given connection. It includes propagation and processing delays.

- Protection Ratio: is defined as the ratio of bandwidth available to a connection after failure to its pre-failure bandwidth allocation.

c) Parameters and Topologies: The performance of the proposed algorithms were studied using a discrete event simulation model built using YACSIM [16]. The network topologies considered were: the 14-node 21-link NSFNET topology (presented in Figure 6(a)) and the 46-node 61-link USANET topology (presented in Figure 6(b)). Each link is bi-directional and is implemented as two uni-directional links.
NSFNET T1 Network 1991

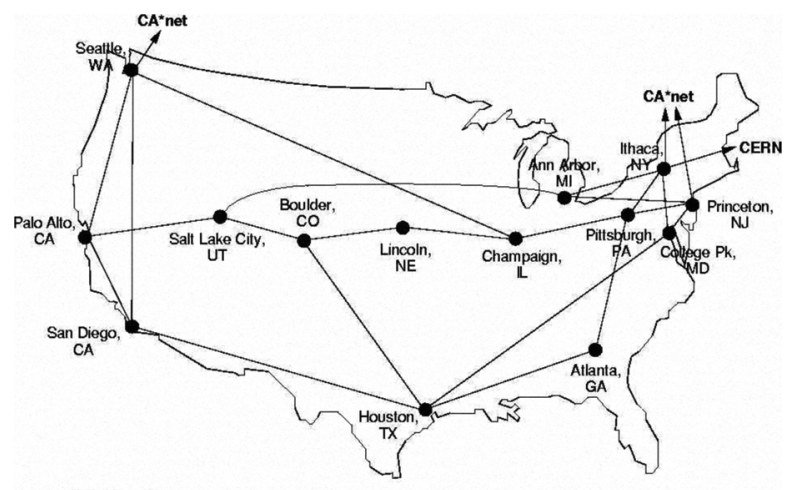

(a) 14-node, 21-link NSFNET

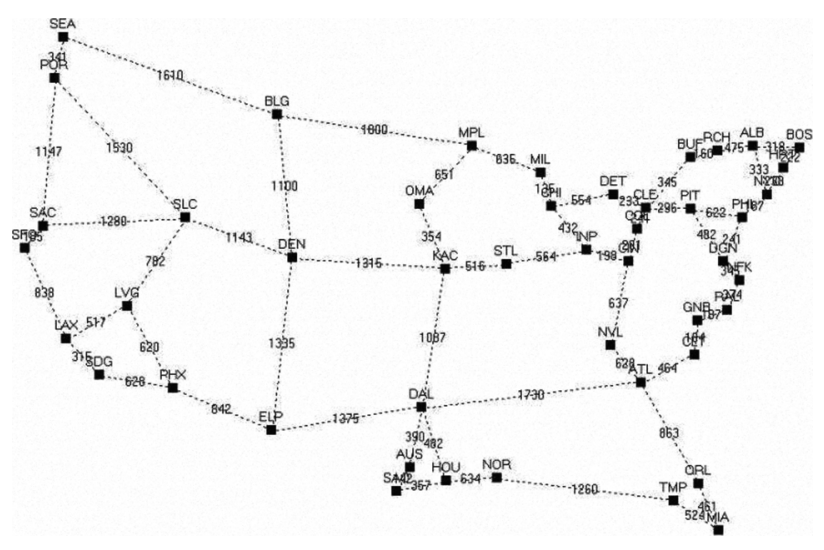

(b) 46-node, 61-link USANET

Fig. 6. Network Topologies Studied.

The system parameters varied are: average connection bandwidth $\left(\mathrm{G}_{a v}\right)$, and connection arrival rate $(\lambda)$. The possible values of $\mathrm{G}_{a v}$ are OC-12, OC-18, OC-24, and OC-30. For each $\mathrm{G}_{a v}, \lambda$ is varied to keep the blocking probability between 0 and 0.1 (i.e. no more than $10 \%$ blocking). In NSFNET, the values of $\lambda$ range from 23 to 30 for OC-18, 18 to 25 for OC24 , and 13 to 20 for OC-30. In USANET, the values of $\lambda$ range from 20 to 27 for $\mathrm{OC}-18,15$ to 22 for OC-24, and 10 to 17 for OC-30.

The simulation results for $\mathrm{G}_{a v}=\mathrm{OC}-12$ are not shown due to extremely low blocking probabilities. For each set of parameter values, simulations were run with different random seeds. Since similar outcomes were observed, only one set of results (instead of the average with confidence intervals) is presented, as a representative sample. SPSW is the traditional route selection method and is used as the base in our performance comparison.

\section{A. Blocking Performance}

Fig. 7 shows that carrying traffic in finer granularity allows a network to admit more connection requests. For NSFNET with $\mathrm{G}_{a v}=$ OC-30, MP, SPMW, and SPMW-MP outperformed 


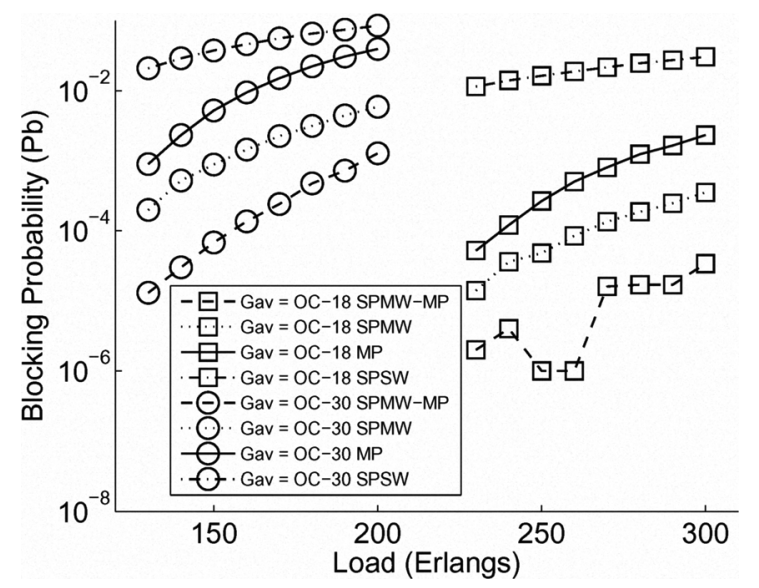

(a) NSFNET

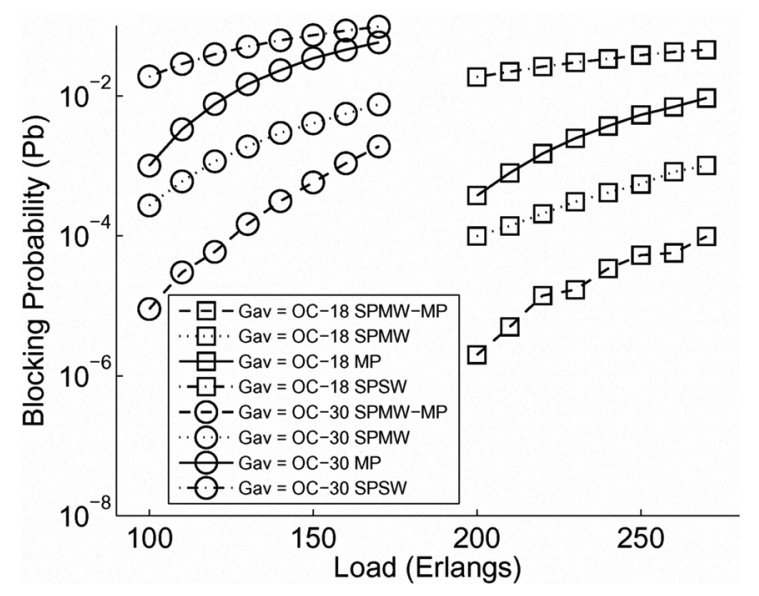

(b) USANET

Fig. 7. Blocking performance vs. load for NSFNET and USANET.

SPSW by $53.4 \%$ to $95.7 \%, 93 \%$ to $99 \%$, and $98.5 \%$ to 99.9\% respectively in terms of blocking performance. For USANET with $\mathrm{G}_{a v}=$ OC-18, MP, SPMW, and SPMW-MP outperformed SPSW by $80 \%$ to $98 \%, 97.8 \%$ to $99.5 \%$, and $99.8 \%$ to $99.9 \%$ respectively in blocking performance. Even though both SPMW and MP carry traffic in finer granularity, SPMW is able to admit more connection requests than MP because SPMW only requires one path. For $\mathrm{G}_{a v}=$ OC-30, the improvement ranges from $78 \%$ to $85 \%$ for NSFNET and $73 \%$ to $87 \%$ for USANET. As expected, SPMW-MP performed better than SPMW in all cases. The dip for SPMW-MP in Fig. 7(a) is a result of extremely small blocking probabilities.

\section{B. Link Utilization}

Fig. 8 shows the average link utilization that correspond to system parameters used Fig. 7. Both SPMW and SPMWMP have slightly higher average link utilization than SPSW because they are able to admit more connection requests. However, MP uses much more link capacity on average compared to SPSW. For $\mathrm{G}_{a v}=\mathrm{OC}-30$ and $\lambda=15$, MP outperformed

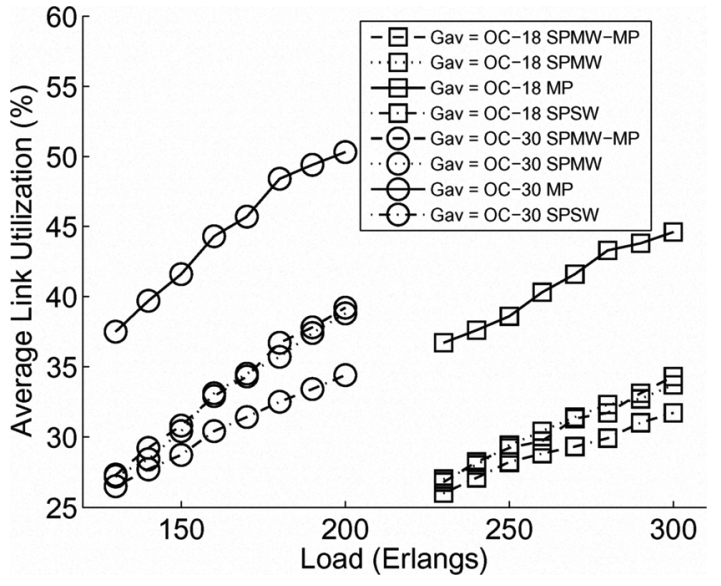

(a) NSFNET

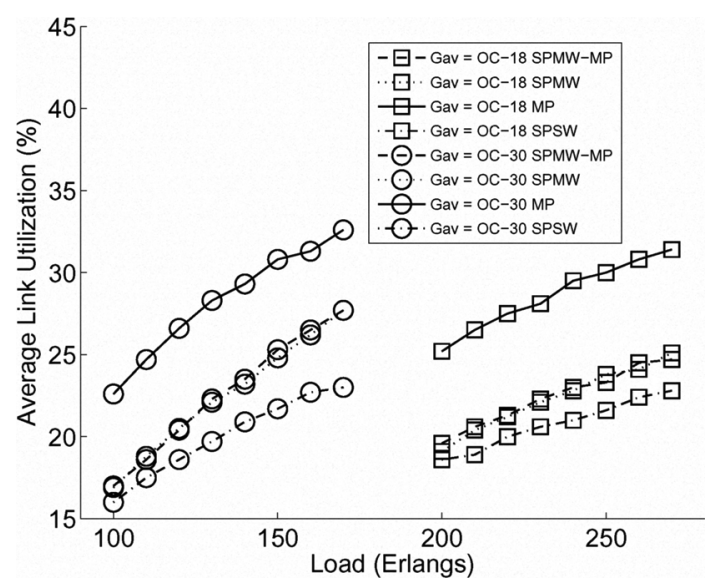

(b) USANET

Fig. 8. Average link utilization vs. load for NSFNET and USANET.

SPSW by $86 \%$ despite $44 \%$ higher link capacity usage with NSFNET, by $53 \%$ despite $42 \%$ higher link capacity usage with USANET. MP utilizes link capacity more efficiently than the traditional SPSW because MP is able to admit more connection requests even though less link capacity is available.

\section{Setup Time}

In a dynamic-traffic network where routing paths are computed on a demand basis, the amount of time needed to establish a connection heavily depends on its number of linkdisjoint paths and the network condition. Fig. 9 shows that the average setup time increases as the number of link-disjoint paths increases. For connections that result in one routing path, their setup time increases with traffic load because less paths are available when the network is congested. For example, assume that the maximum number of link-disjoint paths between $s$ and $d$ of a connection request is 3 . When the network is congested, there is a higher probability that only 1 path is available between $s$ and $d$. Since MP aims to utilize all link-disjoint paths, it will first try to establish the 


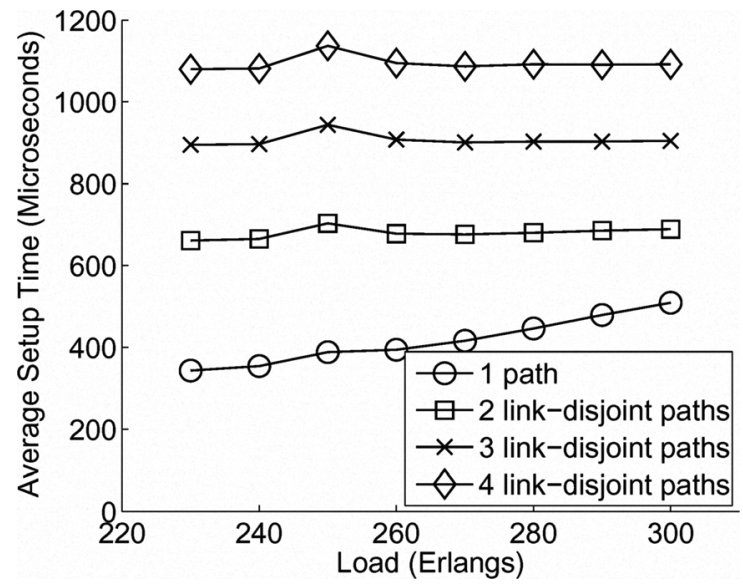

(a) $G_{a v}=O C-18$

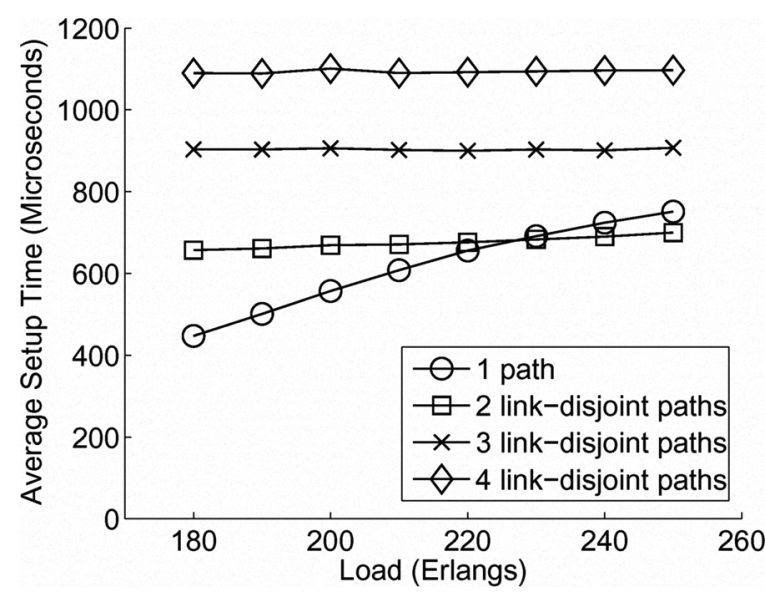

(b) $G_{a v}=O C-24$

Fig. 9. Average setup time vs. load for MP in NSFNET.

connection using 3 paths. If that fails, then it will try using 2 paths. MP will also try using 1 path if the multi-path approach fails. Thus, the average setup time increases with traffic load for single-path connections.

\section{Differential Delay and Link Failure Protection Ratio}

The benefits of routing traffic with multiple link-disjoint paths include efficient link capacity utilization, more evenly distributed traffic load, and implicit partial protection in case of a link failure. However, these benefits come at the cost of differential delay and additional buffer capacity. Average differential delays and their corresponding buffer requirement per link are shown in Table III and in Table IV. These tables also show that a higher implicit link failure protection ratio implies a longer differential delay. The worst case is with NSFNET where 4 link-disjoint paths are used and an average differential delay of 26 milliseconds leads to 130 megabytes (MB) of buffer requirement per uni-directional link. The buffer capacity is calculated as link capacity times average differential delay.
TABLE III

PROTECTION RATIO AND DIFFERENTIAL DELAY FOR MP IN NSFNET.

\begin{tabular}{|l|l|l|l|}
\hline $\begin{array}{l}\text { \# Link- } \\
\text { Disjoint } \\
\text { Paths }\end{array}$ & $\begin{array}{l}\text { Protection } \\
\text { Ratio (\%) }\end{array}$ & $\begin{array}{l}\text { Average } \\
\text { Differential } \\
\text { Delay (ms) }\end{array}$ & $\begin{array}{l}\text { Buffer } \\
\text { Requirement } \\
\text { per Link (MB) }\end{array}$ \\
\hline \hline 2 & 50.0 & 10.1 & 50.0 \\
\hline 3 & 66.7 & 18.4 & 92.0 \\
\hline 4 & 75.0 & 26.0 & 130.0 \\
\hline
\end{tabular}

TABLE IV

PROTECTION RATIO AND DIFFERENTIAL DELAY FOR MP IN USANET.

\begin{tabular}{|l|l|l|l|}
\hline $\begin{array}{l}\text { \# Link- } \\
\text { Disjoint } \\
\text { Paths }\end{array}$ & $\begin{array}{l}\text { Protection } \\
\text { Ratio (\%) }\end{array}$ & $\begin{array}{l}\text { Average } \\
\text { Differential } \\
\text { Delay (ms) }\end{array}$ & $\begin{array}{l}\text { Buffer } \\
\text { Requirement } \\
\text { per Link (MB) }\end{array}$ \\
\hline \hline 2 & 50.0 & 9.7 & 48.5 \\
\hline 3 & 66.7 & 24.1 & 120.5 \\
\hline 4 & 75.0 & 35.8 & 179.0 \\
\hline
\end{tabular}

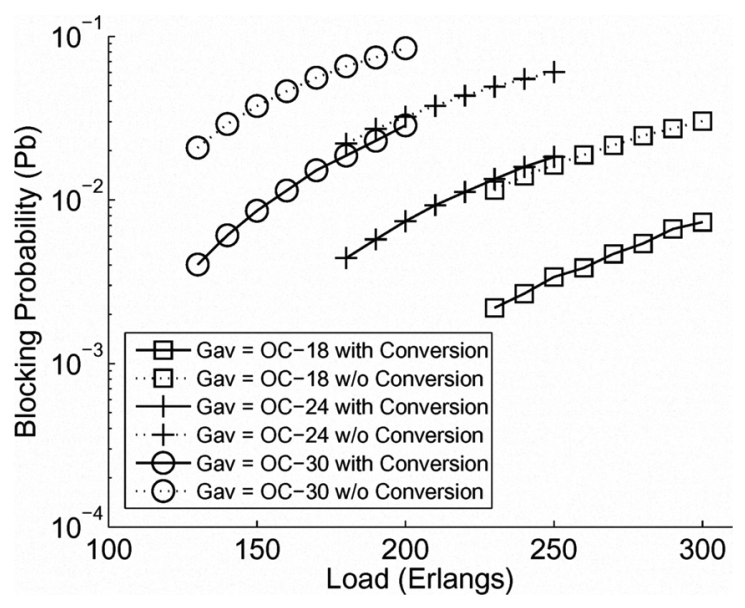

(a) Single-Path

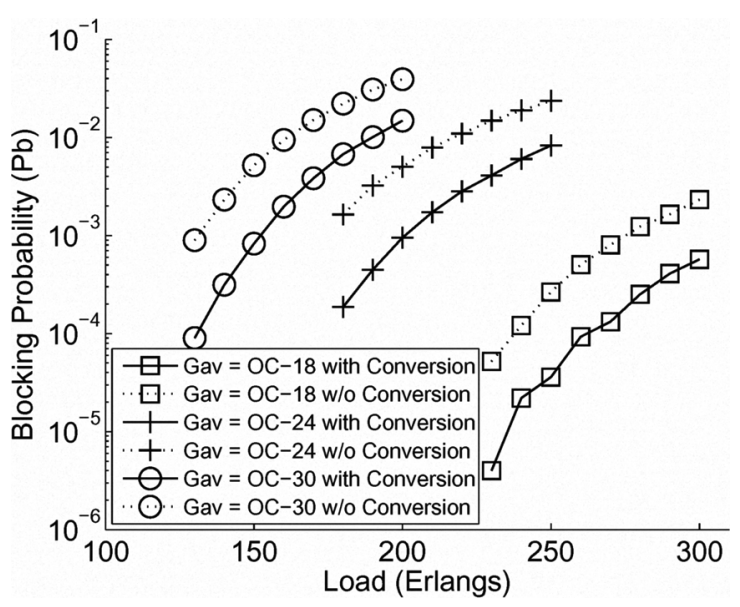

(b) Multi-Path

Fig. 10. NSFNET - Effect of wavelength conversion on blocking performance 


\section{E. Wavelength Conversion}

The above results were done for systems without wavelength conversion. We next present results for networks that have full wavelength conversion enabled. Wavelength conversion allows the use of different wavelengths on a lightpath and reduces the blocking caused by the wavelength continuity constraint. Thus, results shown in Fig 10 for the NSFNET topology, with wavelength conversion, are as expected. For both methods, single-path and multi-path, a significant reduction in blocking probability was observed. The improvement for $\mathrm{G}_{a v}=\mathrm{OC}-24$ ranges from $70 \%$ to $80 \%$ with the single-path method and $65 \%$ to $89 \%$ with the multi-path method. Similar trends are observed for the USANET topology as shown in Fig. 11.

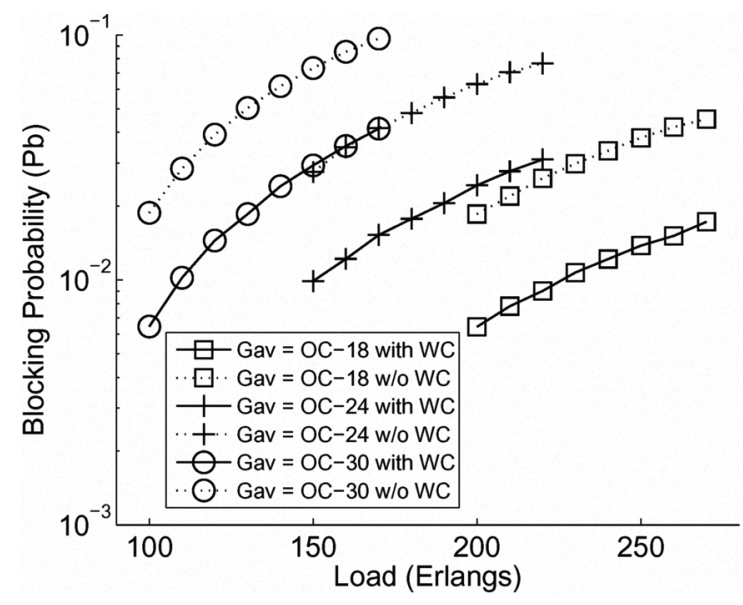

(a) Single-Path

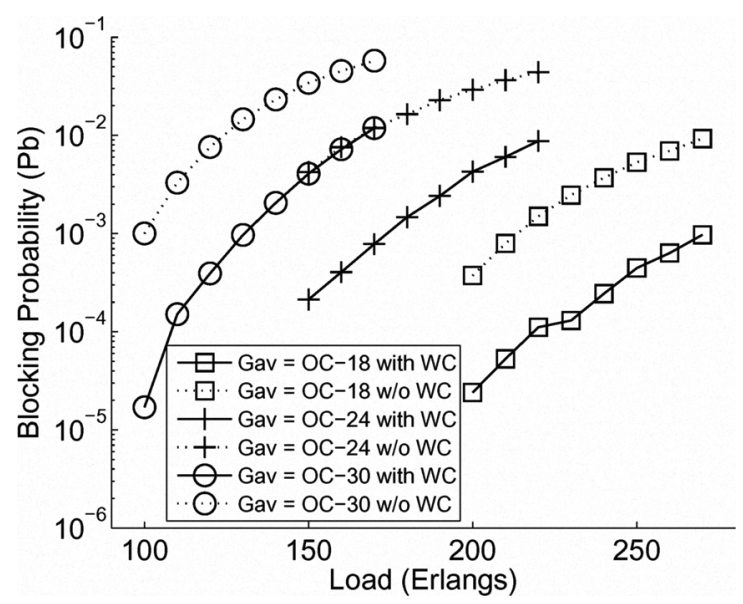

(b) Multi-Path

Fig. 11. USANET - Effect of wavelength conversion on blocking performance

\section{CONCLUSIONS}

In this paper, we investigated problems related to using the VCAT mechanism of SONET technology in optical WDM grooming networks with dynamic traffic demand. We proposed four route selection methods to study the performance of VCAT's traffic splitting feature. The simulation results confirmed the benefits of carrying traffic in finer granularity. Routing connections using multiple paths or a single path with multiple wavelengths can greatly improve a network's blocking performance. When link-disjoint paths are used, connections are also partially protected from link failures. Our study showed that differential delay caused by VCAT's multipath routing increases with the number of link-disjoint paths used for routing. However, with the current cost of memory, it is feasible to provide a sufficient amount of buffer at all receiving nodes to solve the differential delay problem.

\section{ACKNOWLEDGMENTS}

Part of the research was supported by NSF grant No. ANI-0322959; Mr. Kevin Yang was supported by an REU supplement of this grant.

\section{REFERENCES}

[1] K. Sivalingam and S. Subramaniam, eds., Emerging Optical Network Technologies. Boston, MA: Springer Publishers, 2004.

[2] B. Mukherjee, Optical WDM Networks. Springer, 2006.

[3] K. Zhu, B. Mukherjee, and H. Zang, "Exploiting the benefits of virtual concatenation in optical transport networks," in Proc. of IEEE Optical Fiber Communications (OFC) Conference, (Atlanta, GA, USA), Mar. 2003.

[4] L. Choy, "Virtual concatenation tutorial: Enhancing SONET/SDH networks for data transport," Journal of Optical Networking, vol. 1, Jan. 2002.

[5] M. Sivakumar, R. K. Shenai, and K. M. Sivalingam, "A survey of survivability techniques for optical WDM networks," in Emerging Optical Network Technologies (K. Sivalingam and S. Subramaniam, eds.), pp. 297-331, Springer Publishers, 2005.

[6] A. K. Somani, Survivability and Traffic Grooming in WDM Optical Networks. Cambridge University Press, 2006.

[7] W. J. Goralski, SONET/SDH. McGraw-Hill, third ed., 2002. ISBN 0-07-222524-6.

[8] C. Ou, K. Zhu, N. Singhal, and B. Mukherjee, "Survivable Virtual Concatenation for Data-over-SONET/SDH Networks," in Proc. of IEEE Optical Fiber Communications (OFC) Conference, (Los Angeles, CA, USA), Mar. 2004.

[9] S. Ahuja, T. Korkmaz, and M. Krunz, "Minimizing the differential delay for virtually concatenated Ethernet over SONET systems," in Proc. of ICCCN, (Chicago, IL, USA), Oct. 2004.

[10] A. Srivastava, S. Acharya, M. Alicherry, B. Gupta, and P. Risbood, "Differential delay aware routing for ethernet over SONET/SDH," in Proc. IEEE INFOCOM, vol. 2, pp. 1117-1127, March 2005.

[11] A. Srivastava and A. Srivastava, "Flow aware differential delay routing for next-generation ethernet over SONET/SDH," in Proc. IEEE ICC, pp. 140-145, 2006.

[12] S. Huang, B. Mukherjee, and C. Martel, "Survivable multipath provisioning with differential delay constraint in telecom mesh networks," in Proc. of IEEE INFOCOM, pp. 191-195, April 2008.

[13] J. Fang, M. Sivakumar, A. K. Somani, and K. M. Sivalingam, "On partial protection in groomed optical WDM mesh networks," in Proc. of IEEE International Conference on Dependable Systems and Networks (DSN) - Dependable Computing and Communications Symposium (DCCS), (Yokohama, Japan), June 2005

[14] M. Sivakumar, K. M. Sivalingam, and A. Somani, "Partial protection in optical WDM networks: Enhanced support for dynamic traffic," in Proc. of IEEE Communications Society/CreateNet International Conference on Broadband Networks (BroadNets) - Optical Communications and Networking Symposium, (San Jose, CA, USA), Oct. 2006.

[15] S. Rai, O. Deshpande, C. Ou, C. Martel, and B. Mukherjee, "Reliable multipath provisioning for high-capacity backbone mesh networks," IEEE/ACM Transactions on Networking, vol. 15, pp. 803-812, Aug. 2007.

[16] J. R. Jump, YACSIM Reference Manual. Rice University, Department of Electrical and Computer Engineering, 2.1 ed., Mar. 1993. 